\title{
A Hybrid Methodology for Flower Images Segmentation \& Recognition with extended Deep- Convolution Neural Network (CNN)
}

Neeraj Kumar Rathore ( $\nabla$ neerajrathore37@gmail.com )

IGNTU: Indira Gandhi National Tribal University https://orcid.org/0000-0002-9349-3419

Varshali Jaiswal

Rajiv Gandhi Technical University: Rajiv Gandhi Proudyogiki Vishwavidyalaya

Varsha Sharma

Rajiv Gandhi Technical University: Rajiv Gandhi Proudyogiki Vishwavidyalaya

Sunita Varma

Shri Govindram Seksaria Institute of Technology and Science

\section{Research Article}

Keywords: Image segmentation, Deep convolution neural network, Classification, Edge detection, Computer Vision, $L * a * b$ color space

Posted Date: July 12th, 2021

DOl: https://doi.org/10.21203/rs.3.rs-621258/v1

License: (9) This work is licensed under a Creative Commons Attribution 4.0 International License. Read Full License 


\title{
A Hybrid Methodology for Flower Images Segmentation \& Recognition with extended Deep- Convolution Neural Network (CNN)
}

\author{
Neeraj Kumar Rathore ${ }^{1}$, Varshali Jaiswal ${ }^{2}$, Varsha Sharma ${ }^{3}$, Sunita Varma ${ }^{4}$ \\ ${ }^{1}$ Associate Professor, Indira Gandhi National Tribal University (IGNTU)-A Central University, Amarkantak, M.P \\ ${ }^{2}$ Research Scholar, School of Information Technology, RGPV, Bhopal, India \\ 3 Assistant Professor, School of Information Technology, RGPV, Bhopal,India \\ ${ }^{4}$ Professor, Information Technology, Shri. GSITS, Indore, India
}

\{ neerajrathore37@gmail.com,varshalijaiswal@gmail.com,varshasharma@rgtu.net,sunita.varma19@gmail.com\}

\begin{abstract}
Deep-Convolution Neural Network (CNN) is the branch of computer science. Deep Learning CNN is a methodology that teaches computer systems to do what comes naturally to humans. It is a method that learns by example and experience. This is a heuristic-based method to solve computationally exhaustive problems that are not resolved in a polynomial computation time like NP-Hard problems. The purpose of this research is to develop a hybrid methodology for the detection and segmentation of flower images that utilize the extension of the deep CNN. The plant, leaf, and flower image detection are the most challenging issues due to a wide variety of classes, based on their amount of texture, color distinctiveness, shape distinctiveness, and different size. The proposed methodology is implemented in Matlab with deep learning Tool Box and the dataset of flower image is taken
\end{abstract}

from Kaggle with five different classes like daisy, dandelion, rose, tulip, and sunflower. This methodology takes an input of different flower images from data sets, then converts it from RGB (Red, Green, Blue) color model to the $L * a * b$ color model. $L * a * b$ has reduced the effort of image segmentation. The flower image segmentation has been performed by the canny edge detection algorithm which provided better results. The implemented extended deep learning convolution neural network can accurately recognize varieties of flower images. The learning accuracy of the proposed hybrid method is up to $\mathbf{9 8 \%}$ that is maximizing up to $\mathbf{+ 1 . 8 9 \%}$ from state of the art.

Keywords - Image segmentation, Deep convolution neural network, Classification, Edge detection, Computer Vision, $\mathbf{L} * \mathbf{a} * \mathbf{b}$ color space.

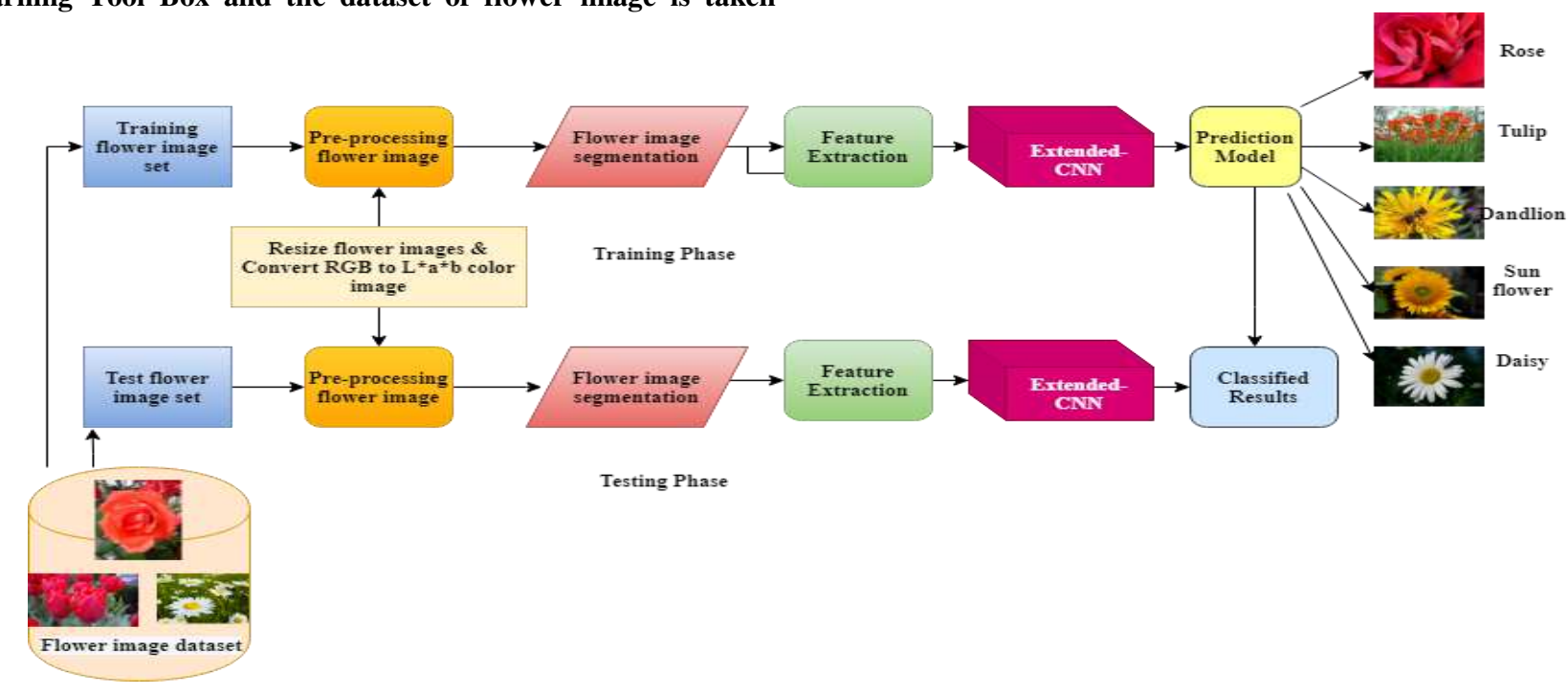

Graphical abstract

\section{INTRODUCTION}

Ayurveda prompts plant, leaf, and flower image segmentation and recognition. Image segmentation refers to separating an image into multiple areas [1]. The objective of segmentation is to make things easy and refine the representation of an image into something that is more expressive and easier to analyze. Image segmentation is normally used to trace the boundaries (lines, curves, etc.) and objects in the image. Edge detection is the segmentation process which identifies the edges of an image. There are various algorithms for edge detection like canny edge detection, watersheds, etc.

The flower image segmentation and recognition are difficult tasks caused by the broad assortment of flower image types, based on their amount of texture, color distinctiveness, shape distinctiveness, and different size. Various categories of the flower image are similar, as shown in figure 1, both in shape or color because of inter-class similarity. 


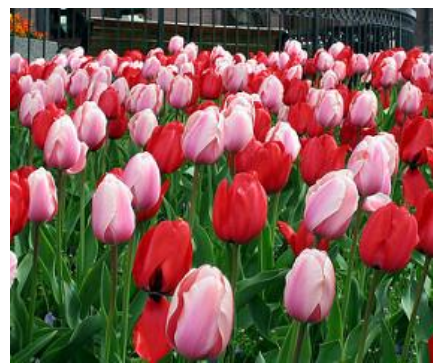

Tulip

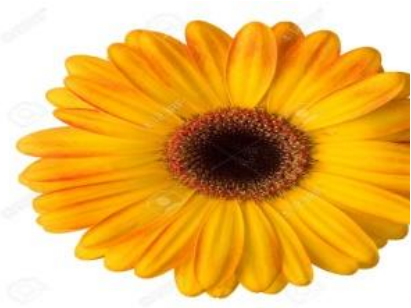

Daisy

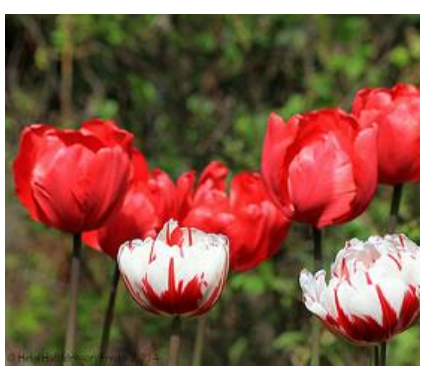

Rose

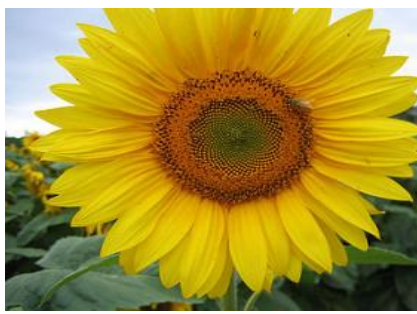

Sunflower
Figure 1: Flower images inter-class similarity.

The deep convolution neural network is used as a tool for image processing to solve various problems [2] such as Object classification, object detection, face recognition, and semantic sub-splitting of the image, etc. In semantic image segmentation, the convolution neural network takes an image as input. After processing the input image it provides output as sub-splitting of the original image where each class is identified with mathematical value and color. The classes are demarcated by the user and speak of the diverse objects in the image like floors, dogs, and chairs. The intermediate layers are associated with learnable weights. The neural network has some significant parameters denoted as hyperparameters. The learning rates, batch size, number of the intermediate layer are some examples of hyperparameters.

Problem Definition - In Ayurveda various types of flowers like rose, bhringraj, hibiscus, etc are used for making medicines. Ayurveda prompts to identify and recognize the flower images. But identifying and recognizing an image is a very difficult task because different species of flowers may look identical in shape as well as in color. It is a very challenging task to maximize accuracy and minimize the error rate of image segmentation and detection of RGB flower images. Similarly, RGB images are considered five-dimensional problems. Since three dimensions are used for red, green, blue. The two dimensions are used for the luminosity layer and chromaticity layer which as solved by $\mathrm{L}^{*} \mathrm{a} * \mathrm{~b}$ color space. It is also considered as a complex computational timeconsuming problem.

To solve this problem proposed system utilize an optimization method of deep convolution neural network for identifying and segmenting flower image and $\mathrm{L} * \mathrm{a} * \mathrm{~b}$ conversion. It is a fast and accurate image segmentation method. In summary, contributions of the proposed research methodology are as follows.

1) Developed and implemented a hybrid method that reads the number of $\mathrm{N}$ different colored flower image from data sets.
2) Every color image has five attributes, first three for colors Red, Green, Blue, and two attributes for the geometry luminosity layer and chromaticity layer. To provide better results and minimize the effort of image segmentation every image is transferred into $\mathrm{L}^{*} \mathrm{a} * \mathrm{~b}$ color [2] [3].

3) Then resultant uniform size flower images are segmented using a canny edge detection algorithm which becomes an essential field of research work to improve the experimental results and identification of various objects in image [4].

4) The segmented flower images are grouped into different cluster. In a cluster, each pixel is similar according to some properties such as color, texture, and intensity.

5) To design an extended deep convolution neural network from resultant image datasets with one hidden layer for prediction of flower images. The deep convolution neural network is structured in computational layers interchanging amongst the convolution layer and max-pooling layer.

6) In the last predicted various flower images with proposed hybrid methodology on flower image datasets.

7) The proposed methodology would be compared with other pre-trained convolution neural networks such as VggNet, GoogLeNet, Alexnet, etc [5].

In this paper a novel extended hybrid deep learning convolution neural network is developed and implemented for flower image detection and segmentation. In the proposed algorithm an RGB image is first converted into $\mathrm{L}^{*}$ $a * b$ image to reduce the efforts in image segmentation and provide effective results. Then an extended deep CNN is trained for detection of flower images with one hidden layer. In the end, the various flower images are recognized by extended deep CNN and the results are calculated. New better flower image segmentation schemes are thus derived, which overcome the problems of conventional systems, by maximizing learning accuracy up to $98 \%$ i.e. increasing $+1.89 \%$ from state of the art and should substantiate augmented flower image segmentation problems.

The remaining sections of the paper are structured as follows: Literature review of research work is highlighted in Section 2. The proposed methodology is illustrated in Section 3. Section 4 shows the experimental setup. Section 5 gives results analysis. In the end, the proposed work is concluded in Section 6 based on the experimental results.

\section{LITERATURE REVIEW}

Flower image detection, segmentation are essential and challenging task in Ayurveda. Ayurveda uses various types of flower like Marigold, Hibiscus, Sunflower, Lavender, Echinacea, Chamomile, Yarrow, Cornflower, Rose, Bhringraj, etc in medicines. But it is a difficult task as the different species of flowers may look very identical, both in shape or color inter-class.

Many researchers are working on flower image detection and segmentation. Table 1. gives a comparison study about related works with performance criteria. 
TABLE I

Row Authors $\begin{gathered}\text { Location of } \\ \text { study }\end{gathered}$ Type of data sets $\quad \begin{gathered}\text { Date of } \\ \text { researc }\end{gathered}$

$$
\text { Image }
$$

Kristen et al. [11]

USA

Datasets/Caltech10

2005

Jonathan Long

[17]

UC Berkeley

PASCAL VOC

2011 and 2012

ImageNet Large-

2011

Scale Visual

Krizhevsky [15]

Toronto

Recognition

2012

(ILSVRC)

Jeff Donahue

Karen Simonya

et al. [23]

Ross Girshick

[13]

Qing Li et al.

[18]

canada

(ILSVRC)

2014

ILSVRC-2012

dataset

2014

UC Berkeley V VOC 2007 dataset 2014

United States

ILD database

2014

GoogLeNet

ensemble

ILSVRC-2012

2015

Karen Simonya

$$
\text { et al. }
$$

UK

Xiaofeng Zhu et

$$
\text { al. [25] }
$$

dataset

2015

MCI-NC 2015

Kaiming He et al. [22]

USA and

Korea ILSVRC \& COCO
2015

2015

2015

2016

Hayit Greenspan et al. [14]

USA

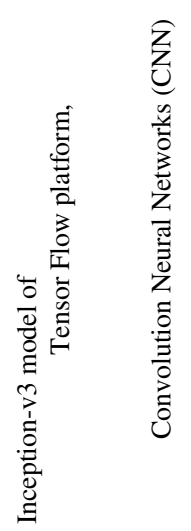

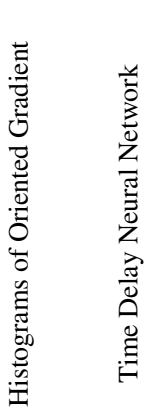

USA

Qi Dou et al. [16]

Ling Zhang et al. [21]

Hong Kong

SWI-CMB

2016

China

Herlev Dataset

2016

Oxford- 102

flower.

Oxford-I7 flower dataset

China

FLOWERS17

FLOWERS102

Visual Geometry

group at University

$$
\text { Oxford } 17
$$

2018

PSREU, USA

2019

Kaggle datasets

2020

(Continued) 


$$
\| 111111111
$$

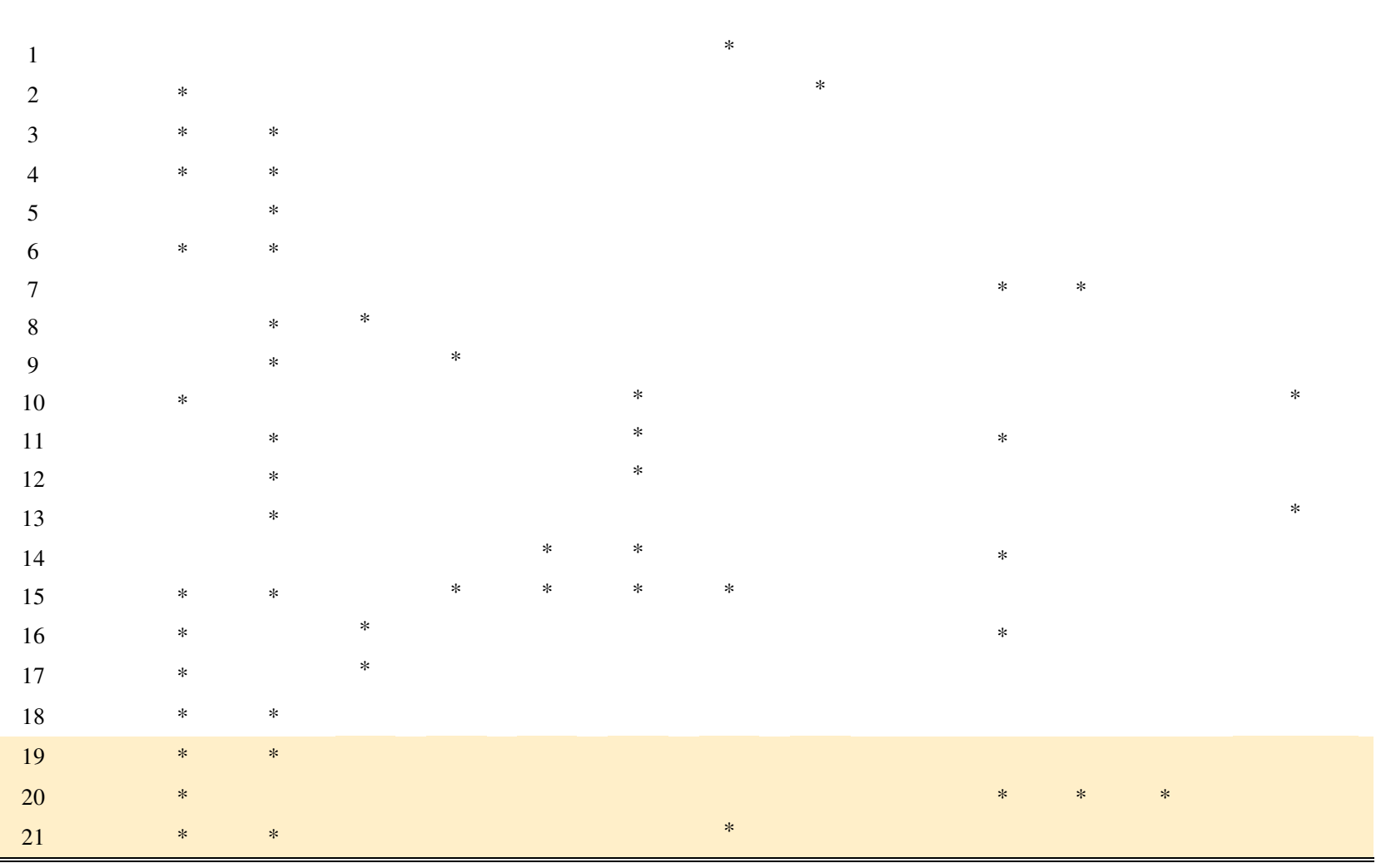

This section reviews some state of arts in the area of flower image segmentation. Hazem et al. proposed a flower image segmentation, classification, and detection method using the deep learning method [6]. This approach tested the three well-known datasets and outcomes were equated with supplementary methods. The accuracy of the proposed methods is up to $97 \%$. The proposed approach of flower images segmentation used localization of the minimum bounding box around images and then utilized binary classification in a convolution neural network for detection of flower images.

Rashmi et al. proposed a new architecture of the convolution neural network to implement mobile Net applications for the classification of flower images by reducing the size and latency of original images. The application was small, efficient, and optimized the results [7]. The experiment was examined on the TensorFlow platform and results shows system required minimize time and space of flower images classification.

A flower classification approach proposed by Xiaoling et al. used the Inception-v3 model of the TensorFlow platform
[8]. This flower image classification technique utilized the transfer learning method. The accuracy of flower image classification was up to $95 \%$ on Oxford 17datasets and $94 \%$ on 120 ' flower datasets.

A new Convolution Neural Network approach proposed by I.Gogul et al. trained the small data sets and utilized general purpose computation resources [9] the approach classified The flower image into three different parts and then extracted the features of flower images by using Over Feat CNN in training data sets. The proposed system utilized machine learning techniques for training a neural network.

This proposed system had accuracy up to Rank-1, $82.32 \%$ and Rank-5 accuracy of $97.5 \%$ using machine learning classification FLOWERS 28 dataset [10]. The researchers proposed a flower recognition approach with Support Vector Machine classification [11]. This approach used natural flower images for image segmentation purpose and learning accuracy of the system was up to $85.93 \%$.

\subsection{Image Segmentation}

The image segmentation method improves the performance of object recognition and identification. Image segmentation is a technique of partitioning an image into a segment that is similar according to sets of predefined criteria. Semantic 
segmentation faces an inherent tension between semantics and location [12]. Region-based classification is a standard technique for semantic segmentation that can be easily applied on flower image segmentation [13]. Flower image segmentation is the technique of detecting edges of flower image that make into a different group. Pixels or spots in a group are similar according to some properties of a group such as color, texture, and intensity.

\section{$1.2 L * a * b$ color Space}

The color model based on $\mathrm{L}^{*} \mathrm{a} * \mathrm{~b}$ space is q layered design method in which the red-green axis denoted by chromaticity layer 'a*'. Similarly, blue-yellow axis is denoted by chromaticity layer ' $b$ * and luminosity is denoted by ' $\mathrm{L}^{*}$ ' layer. The color image has five attributes, first three attributes for the color red, green, blue, and two attributes for the geometry luminosity layer and chromaticity layer. To provide a better result and minimize the effort of image segmentation, every image is transferred into $\mathrm{L}^{*} \mathrm{a} * \mathrm{~b}$ color model. The conversion between the RGB color model and the $\mathrm{L}^{*} \mathrm{a} * \mathrm{~b} *$ color model is not a straight forward process.

\subsection{Artificial Deep learning Convolution Neural Network}

The biological neural network is the inspiration of convolution neural network [14]. Artificial deep learningbased convolution neural network is a mathematical model. Mathematical models are weighted function, input function and transform function [15]. Figure 2 represents the architecture of artificial deep convolution neural network, where $\mathrm{R}$ is size of input and corresponding output.

$$
\mathrm{n}=\mathrm{w}_{1,1} \mathrm{p}_{1+} \mathrm{w}_{1,2} \mathrm{p}_{2+\ldots .} \mathrm{w}_{1, \mathrm{R}} \mathrm{p}_{\mathrm{R}+\mathrm{b}}
$$

Where $\mathrm{f}$ represents mathematical model, a represented activation function, $w$ represents a weight function, $b$ represents a biased value, and $\mathrm{p}$ represents input value.

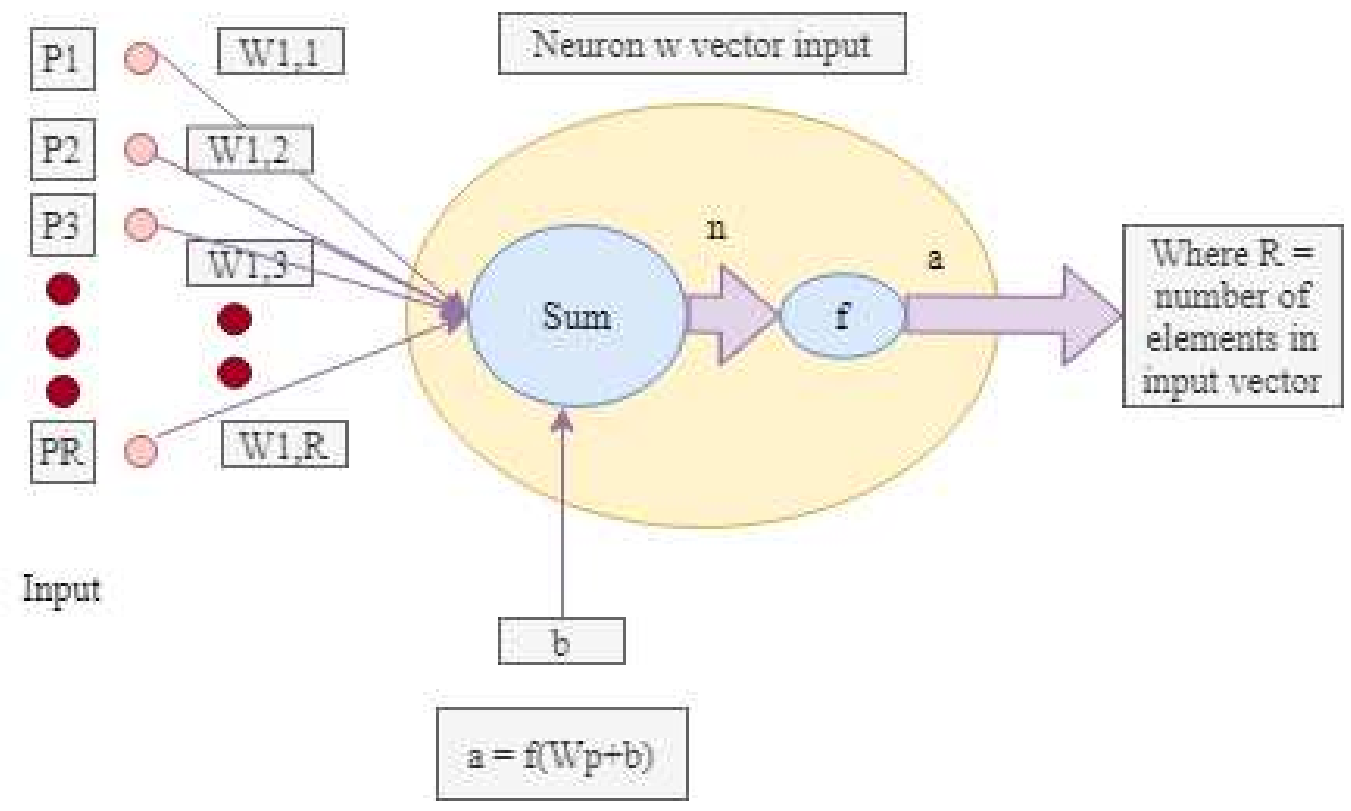

Figure 2: Architecture of artificial deep learning-based convolution neural network.

Researchers researched in the area of deep learning for recognizing and segmentation of images. Pheng et al. presented an improved technique to automatically identify cerebral microbleeds from magnetic resonance volumes, leveraging $3 \mathrm{D}$ convolution neural network [16]. The proposed system utilized a 3D convolution neural network for recognition of cerebral microbleeds for magnetic resonance images. The authors compared experiment results with previous method that was based on the $2 \mathrm{D}$ convolution neural network. The proposed method had enhanced recognition accuracy compared to other methods. This method used for image recognition and sub-splitting tasks with an advanced application of a 3D convolution neural network for volumetric medical datasets.

The authors Jonathan et al. implemented fully convolution neural networks for semantic segmentation problem and improved the architecture with a multi-resolution layer [17]. Fully convolution neural networks take input of arbitrary size and produce output with the corresponding size. The authors utilized Alex Net, VGG Net, and GoogleLeNet with fully convolution neural networks to generate efficient and meticulous semantic segmentation of images. This combination dramatically improved the state-of-the-art, while simultaneously simplified and speeded the learning rate and inference.

Qing et al. proposed a customized convolution neural network architecture to categorize HRCT lung picture patch of ILD pattern [18]. The system designed a convolution neural networks with a single layer that learns the DCT pattern from training samples capably and produced efficient classification results. The experiment results show that the proposed approach is capable of extracting discriminative features automatically and achieve efficient target performance. In the experiment observed that the limited size of training data and blurry structure were complicated in adjusting the convolution neural network to ILD image classification.

Deep convolution neural networks were produces efficient results in the field of image recognition, categorization with a large size of data sets [19] [20]. Several researchers are working to achieve efficient classification results or 
recognition of objects accurately without considering the computation complexity [21] [22] [23]. The additional hardware requirement of the convolution neural network is graphics processing units or titanium processor to process large datasets.

\subsection{Classification}

Classification of flower image is a challenging task due to the intra-class variability with different lighting conditions. The proposed flower image segmentation method used a binary classification technique to detect flower images. The use of extended deep convolution neural networks [5] is for classification problems where the output to an image is an alone class label [24]. However, in many computer vision problems, particularly in biomedical image processing the desired output should include localization [25] [26].

It is similar to flower image classification because of varieties of flower images, having similarities in various groups. Satoshi et al. implemented a color classification of an object that utilized three heterogeneous co-occurrence colors such as CoHD, CoHOG, CoHED [27]. The results produced by that method efficiently achieve a high classification rate due to high dimensional and highly discriminating co-occurrence features.

\section{Proposed METHOdOLOGY}

This section proposed, a hybrid methodology for identifying and splitting up of flower image using the $L * a * b$ color space with an extended deep convolution neural network. The improvement of artificial neural networks is done by a deep learning approach. Deep learning having supplementary layers that authority higher levels of abstraction and increasing objects detection rate. Figure 3 describes the proposed hybrid methodology for flower image segmentation and recognition with $\mathrm{L} * \mathrm{a} * \mathrm{~b}$ color space by an extended deep convolution neural network. The proposed algorithm work as follows:

Step 1: $\mathrm{N}$ number of different flower images are read from the data sets in which each image has five attributes, three attributes of color ( Red, Green and Blue) and two attributes for geometry (luminosity layer and chromaticity layer). The conversion between RGB to $\mathrm{L}^{*} \mathrm{a} * \mathrm{~b}$ color space [3] improved the performance of image segmentation and produced excellent results.

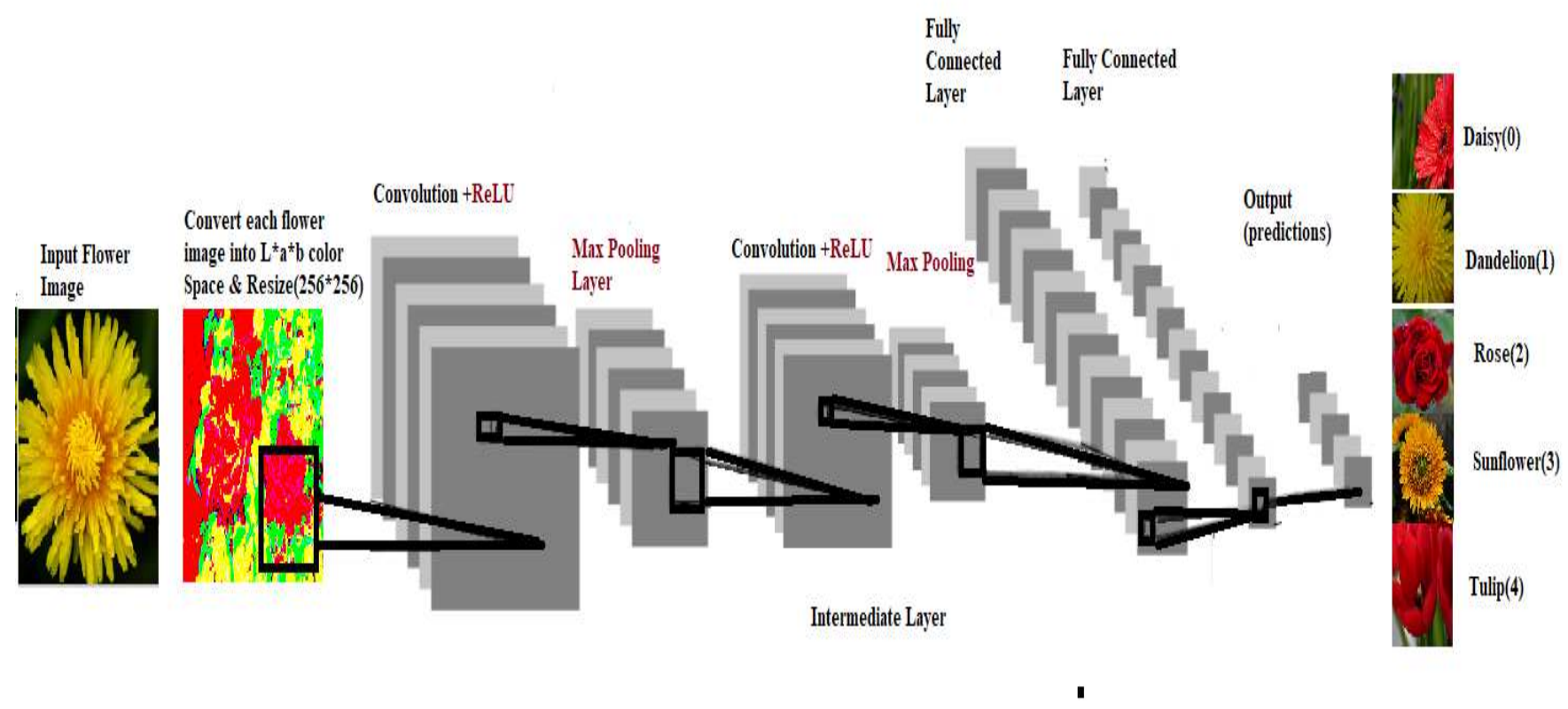

Figure 3: A deep convolution neural network architecture that represents three layers of abstraction with the parameter value.

Step 2: Then resultant uniform size flower images are segmented using a canny edge detection approach which becomes an essential field of research work to improve the experimental results and identify various objects in images. Flower image segmentation is the technique of detecting edges of flower images that make into different groups. Pixels or spots in a group are similar according to some properties of a group such as color, texture, and intensity. Figure 4 shows below is results of conversion between RGB to $\mathrm{L}^{*} \mathrm{a} * \mathrm{~b}$ color space with canny edge detection flower

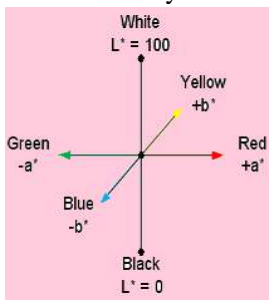

$\mathrm{L}^{*} \mathrm{a} * \mathrm{~b}$ color space images

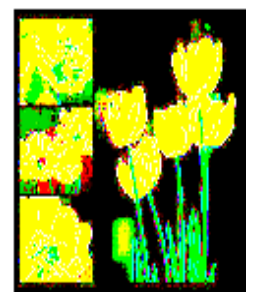

Tulip

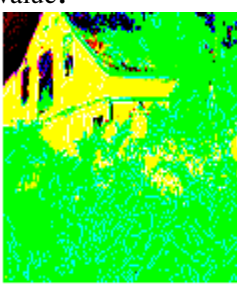

Daisy image segmentation downloaded from the experiment. 


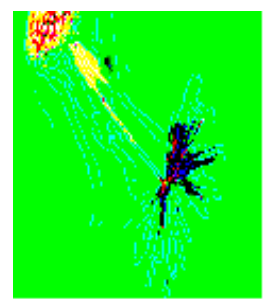

Dandelion

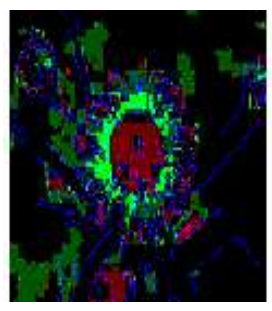

Sunflower

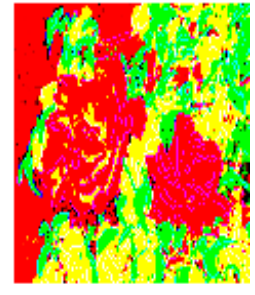

Rose
Figure 4: Different L*a*b flower color space segmented images.

Step 3: An extended deep convolution neural network is then trained from flower image data sets. There are various ways to modify the deep convolution neural network like altering the input image type, sliding stride, filter size, and filter types. In the proposed methodology modified input layer. The input layer reads $\mathrm{L}^{*} \mathrm{a} * \mathrm{~b}$ image instead of the RGB image for learning.

1) The first image input layer, grips the processed pixel value of the image in which image is represented by height and width with dimensions of the color for normalization of an input image.

2) The second convolution layer calculates the results of neurons that are associated with restricted areas in the participation. Each node calculated scalar multiplication between their weights and input value which are associated with the input region.

These may consequence in size and figure 5 represents the convolution layer. This figure is taken an input array of a pixel with different kernels size and generates an output pixel in the form of an array.

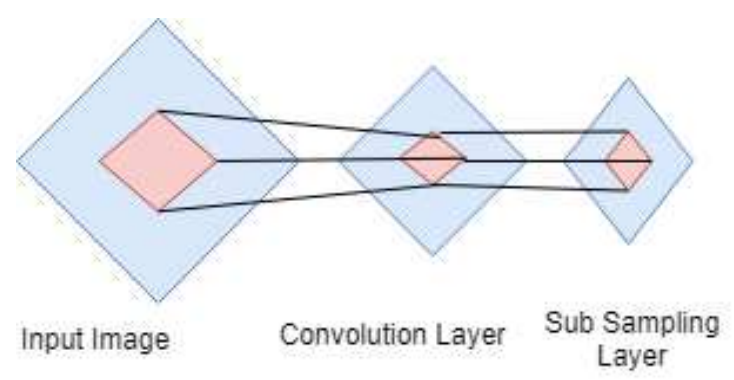

Figure 5: Convolution layers.

Layer of a fully connected convolution neural network described by equation 2 :

$z=\sigma(W x+b)$

Here, $\mathrm{x}$ represents input vector, $\mathrm{z}$ represents output vector, $\mathrm{W}$ represents weight matrix, b represents bias vector and $\boldsymbol{\sigma}$ (q) represents activation function. The figure 6 described working on convolution layer which is the output of dot product of two matrixes: one is image matrix and the second is filtered (kernel) matrix with different load values [28].

\begin{tabular}{|l|l|l|}
\hline 1 & 0 & 1 \\
\hline 0 & 1 & 0 \\
\hline 1 & 0 & 1 \\
\hline \multicolumn{3}{|c|}{ Filter } \\
\hline
\end{tabular}

\begin{tabular}{|l|l|l|l|l|}
\hline 1 & 1 & 1 & 1 & 0 \\
\hline 0 & 1 & 1 & 1 & 0 \\
\hline 0 & 0 & 1 & 0 & 1 \\
\hline 1 & 1 & 0 & 1 & 0 \\
\hline 1 & 1 & 0 & 1 & 1 \\
\hline
\end{tabular}

Image

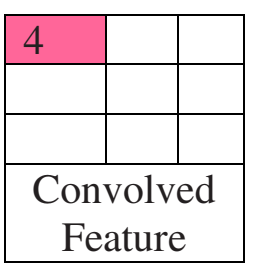

Figure 6: Dot products of matrix used by convolution layer.
For example, a convolved feature value equal to 4 is calculated by the dot product of the filter matrix and image matrix, $1 * 1+0 * 0+1 * 0+0 * 1+1 * 1+0 * 0+1 * 1+0 * 1+1 * 1=4$ and the same calculation is repeated for all regions. Convolution layer is related to convolution of two signals represented by the equation 3 :

$$
f 1[x, y] * f 2[x, y]=\sum_{n 1 \rightarrow-\infty}^{\infty} \sum_{n 2 \rightarrow-\infty}^{\infty} f 1[n 1, n 2] * f 2[x-n 1, y-n 2]
$$

3) The threshold operation is performed by third layer called ReLU layer. In ReLU layer, any value of weight less than zero are assigned to zero.

$$
\begin{aligned}
f(x) & = \begin{cases}x, & x \geq 0 \\
0, & x<0\end{cases} \\
\mathrm{f}(\text { net }) & =\max (0, \text { net })
\end{aligned}
$$

The ReLU layer not changed the size of volume that is and produces an activation function that is $\max (0, \mathrm{x})$ with a threshold value at zero. Equation 3 show activation function with input value $\mathrm{x}$ and corresponding output function $\mathrm{f}$. ReLU considered as the nonlinearity in the convolution neural network.

Dimensions of output volume $=\frac{[W-F+2 P]}{S}+1$

The dimensions of output volume represented by equation 5 , in which $\mathrm{W}$ represented input volume, $\mathrm{F}$ represented size of the filter, where $\mathrm{P}$ represented the amount of padding, and $\mathrm{S}$ represented the stride. Figure 7 show ReLu layer used by the proposed method.

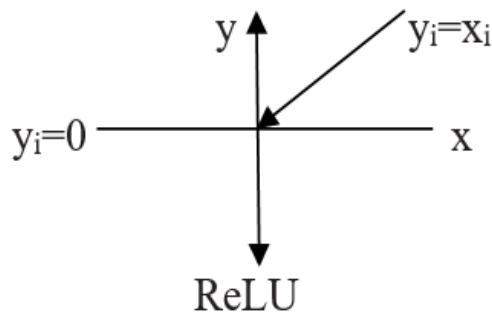

Figure 7: ReLu layer used by proposed method.

4) Fourth Max-pooling layer accomplishes a behind sample process alongside the spatial dimension height and width consequential in a dimension similar to [16x16x12]. Likewise, if pool-Size is $[2,3]$, then the maxpool layer calculates average value of region of height 2 and width 3 . 
Figure 8 shows a matrix representation of maxpooling layer in the convolution neural network.

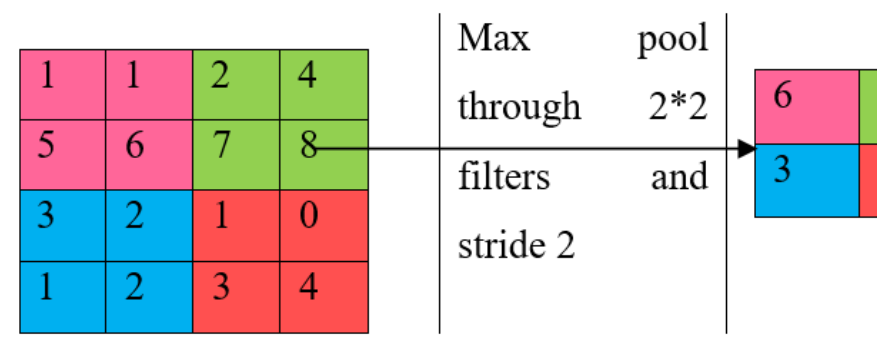

Figure 8: Matrix representation of maxpooling layer in the convolution neural network.

Size of output dimension $=\frac{[\mathrm{W}-\mathrm{F}]}{\mathrm{S}}+1$

5) In the proposed method used one intermediate layer (hidden layer) which is again a combination of the entire above layer.

6) The fifth layer is a fully-connected layer in which those neurons are considered that are fully connected in the Maxpooling layer. It is a fully connected layer for convolution neural network with activation function like Soft-Max for various classification approaches. It produces resultant output with dimension, volume $[1 * 1 * \mathrm{~N}]$ where $\mathrm{N}$ represents the number of classes for classification.
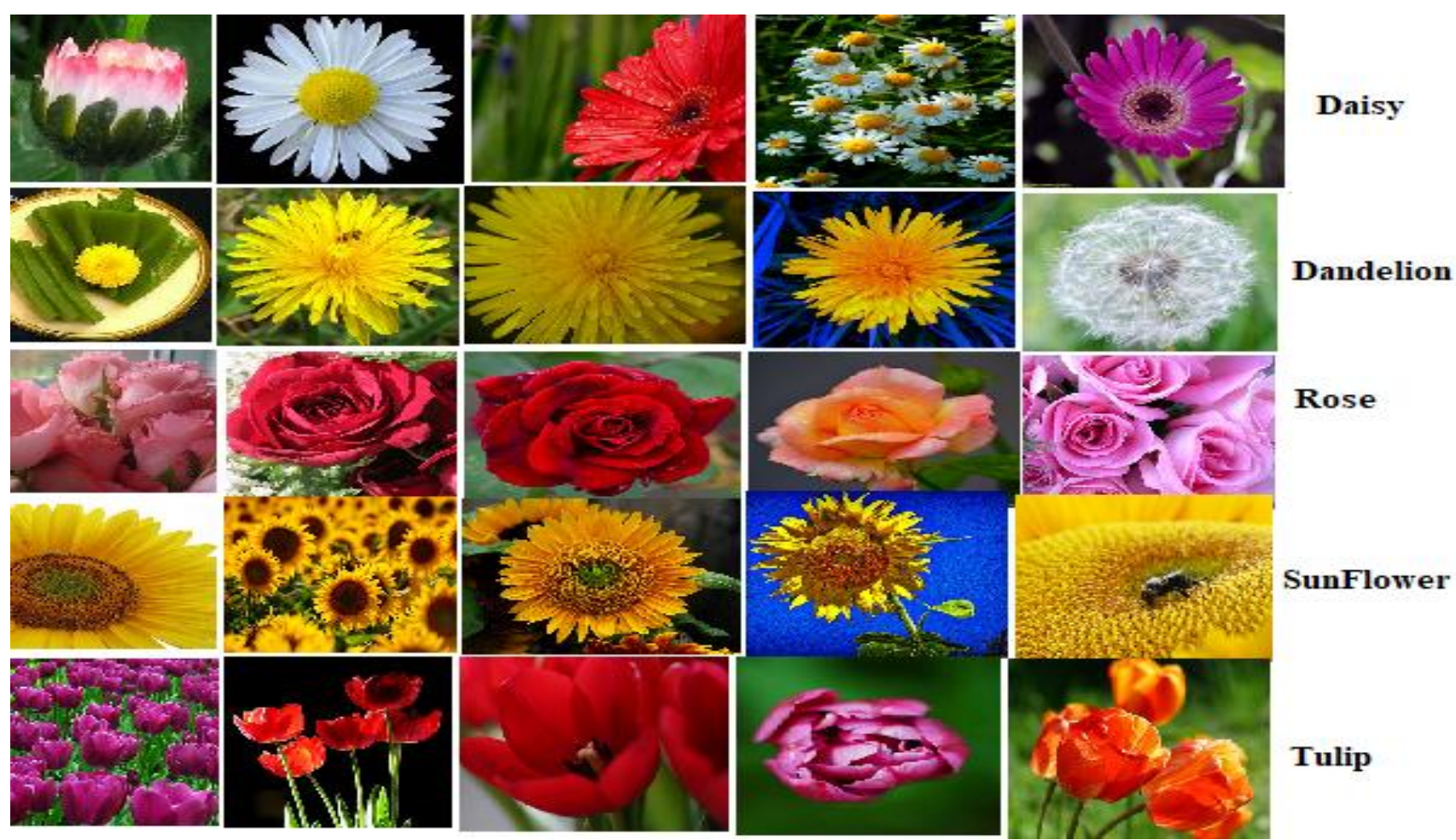

The proposed method is simulated and results are analyzed for the publicly available five flower families taken from Kaggle. The five flower families are Daisy, Dandelion, Rose, Sunflower, and Tulip. The proposed extended hybrid methodology is experimented with coloured flower images from Kaggle's flower image data sets with size of $512 * 512$ pixels [30]. In this experiment system uses $80 \%$ images for training and $20 \%$ images for testing.The Figure 9 shows some flower images from different families of flowers from Kaggle's flower image data sets.

Figure 9: Flower data set used to train the flower identification

\subsection{Evaluation Metrics}

There are a number of parameters used to calculate the accuracy of flower image classification, segmentation, and detection in the suggested method.
Accuracy $=\frac{\operatorname{Sum}(Y \text { Test }==T \text { Test })}{\text { Numel }(\text { T Test })}$

Error rate $=1-$ Accuracy $=\frac{\operatorname{Sum}(Y \text { Test }==T \text { Test })}{\text { Numel }(\text { T Test })}$

Accuracy is measured as the ratio of the count of accurate labels inside the test flower image datasets corresponding to 
the classification from classify, to the count of images in the test flower images.
Error rate defined as one minus accuracy if maximize accuracy then minimizes the error rate.

1.6 Pseudo code

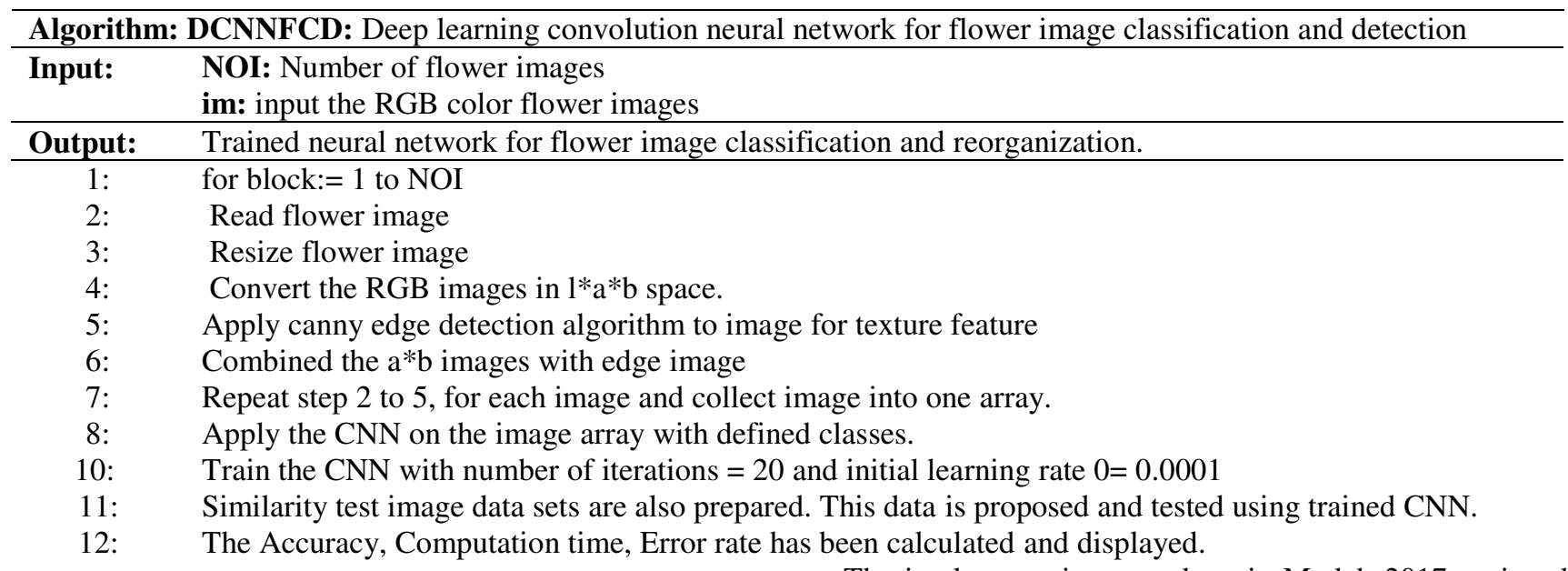

\subsection{Implementation Details}

The proposed system experimented in two different sections, Experiment_1 and Experiment_2 with different hardware configurations and the different number of processed images. In Experiment_1, minimum hardware configuration and only $100+$ images were used whereas in

Experiment_2 extra hardware GPU with 32GB RAM and 1000+ images were used.

\section{Experiment_1}

In this experiment, 5 to 20 different images, each of five flower families (Daisy, Dandelion, Rose, Sunflower, and Tulip) were used. During the experiment_1, 100 images were used by the proposed method from the database.

\section{Implementation Details for Experiment 1}

TABLE II

DETAILED CONFIGURATION OF THE PROPOSED METHOD

\begin{tabular}{lll}
\hline \hline S.NO & Layer & Parameter \\
1 & Convolution Layer $1-2$ & 5,20, stride $=1$ \\
2 & Max pool & 3, stride $=2$ \\
3 & Convolution Layer 3-4 & $3 * 3,64$, stride $=1$ \\
4 & Max pool & $2 * 2$, stride $=2$ \\
5 & Fully Connected Layer & $5, / /$ output size \\
\hline \hline
\end{tabular}

In experiment 2, 165 different images of each type of flowers used, so that total of 825 images was processed in the database.

\section{Implementation Details for Experiment 2}

Experiment_2

The implementation for experiment_2 was done in Matlab 2019a using deep learning Tool Box and results were calculated on an Intel(R) Xeon(R) W-2133 cpu@3.60GHz 
32.0 GB RAM running window 10 Pro for Workstations and 64-bit Operating System, x64-based processor. Simulation parameter used by the experiment- 2 was same as experiment_1 which is shown in Table 2. The flower image segmentation and classification utilizes extended convolution neural network in which first all images are resized to $64 \times 64 \times 3$ to provide a uniform and normalized combination of images that pass through the convolution neural networks.

NOI (Number of images of each class $)=165$ and Training on single GPU.

Initializing input data normalization.

\section{RESULTS ANALYSIS}

\section{Results Analysis of Experiment 1}

Experiments were conducted over downloaded flower image datasets from the website Kaggle. In Table 3 the first column shows the NOI (number of images of each type of flower and there are five types of flower used by the proposed algorithm for simulation), the second column represents epoch, and the following columns represent the time elapsed, loss, accuracy, and learning rate respectively, used by the proposed algorithm. The training was done on a single CPU by initializing flower image normalization by $256 * 256 * 3$.

TABLE III

PERFORMANCE OF THE PROPOSED METHOD

\begin{tabular}{|c|c|c|c|c|c|}
\hline NOI & Epoch & Time Elapsed (Seconds) & $\begin{array}{l}\text { Mini-batch } \\
\text { Loss }\end{array}$ & $\begin{array}{l}\text { Mini-batch } \\
\text { Accuracy }\end{array}$ & $\begin{array}{l}\text { Base-Learning } \\
\text { Rate }\end{array}$ \\
\hline \multirow[t]{2}{*}{4} & 1 & 8.00 & 6.2823 & $15.00 \%$ & $1.00 \mathrm{e}-04$ \\
\hline & 20 & 137.57 & 0.0002 & $100.00 \%$ & $1.00 \mathrm{e}-04$ \\
\hline \multirow[t]{2}{*}{8} & 1 & 13.61 & 7.8754 & $20.00 \%$ & $1.00 \mathrm{e}-04$ \\
\hline & 20 & 266.59 & 0.0353 & $99.00 \%$ & $1.00 \mathrm{e}-04$ \\
\hline \multirow[t]{2}{*}{12} & 1 & 20.45 & 6.0824 & $21.67 \%$ & $1.00 \mathrm{e}-04$ \\
\hline & 20 & 404.36 & 0.0383 & $98.73 \%$ & $1.00 \mathrm{e}-04$ \\
\hline \multirow[t]{2}{*}{16} & 1 & 28.53 & 6.9970 & $23.75 \%$ & $1.00 \mathrm{e}-04$ \\
\hline & 20 & 533.66 & 0.0409 & $98.35 \%$ & $1.00 \mathrm{e}-04$ \\
\hline \multirow[t]{2}{*}{20} & 1 & 62.21 & 7.7679 & $18.00 \%$ & $1.00 \mathrm{e}-04$ \\
\hline & 20 & 711.51 & 0.7370 & $97.75 \%$ & $1.00 \mathrm{e}-04$ \\
\hline \multirow[t]{2}{*}{24} & 1 & 126.00 & 6.4503 & $22.50 \%$ & $1.00 \mathrm{e}-04$ \\
\hline & 20 & 6249.48 & 0.9507 & $95.00 \%$ & $1.00 \mathrm{e}-04$ \\
\hline
\end{tabular}

The proposed algorithm was experimented with different number of flower images and maximum epoch was 20. From the results, proposed method shows that when NOI = 4 and epoch is equal to 20 , then the time required for training is $137.57 \mathrm{~s}$ and accuracy is $100 \%$ with minimum batch loss equal to 0.0002 and learning rate is $1.00 \mathrm{e}-04$.
Figure 10 gives an average of the computation time in seconds taken by over the proposed algorithm for training flower image with NOI (number of images). But when $\mathrm{NOI}=20$, total images $=40$, then at epoch $=20$, the time required for learning rate is $266.59 \mathrm{~s}$ and accuracy decreased only $95.00 \%$ with loss 0.1353 . 


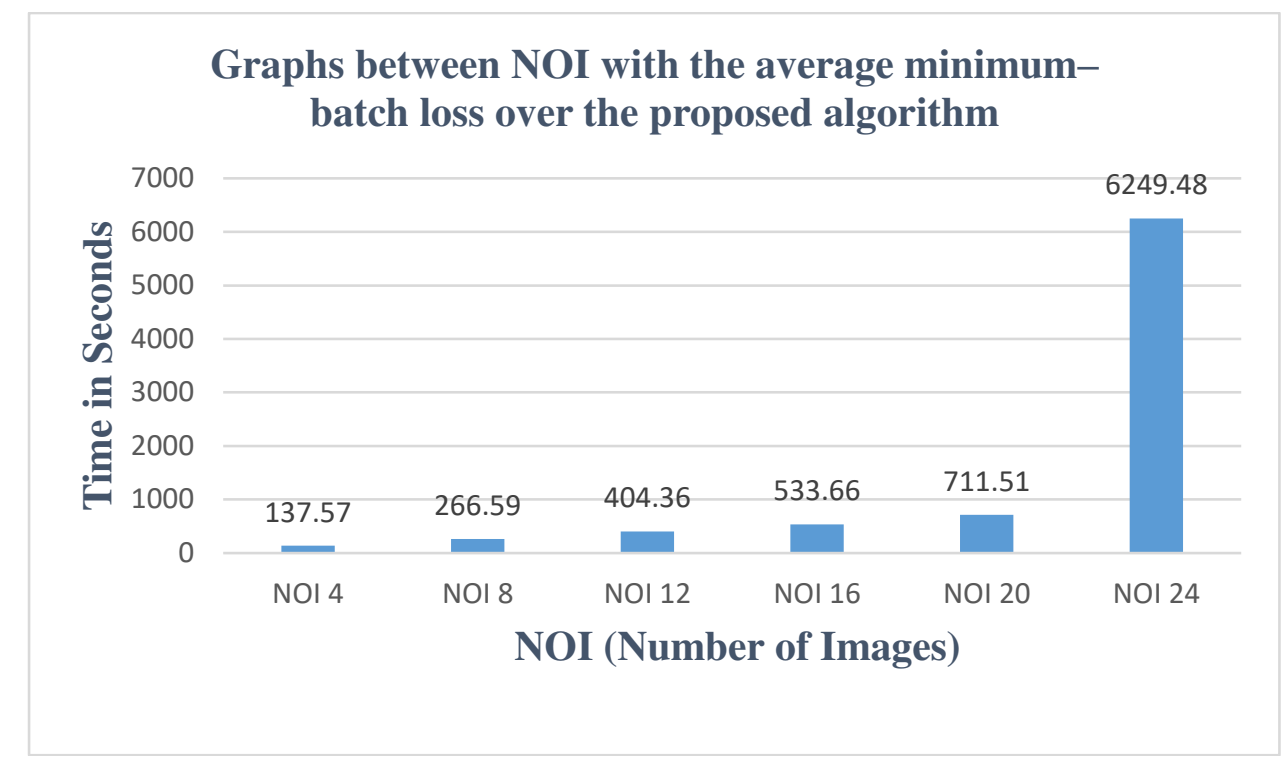

Figure 10: The Average of the computation time in seconds taken by the proposed algorithm for training flower image with NOI (number of images).

Again, when NOI $=12,16,20,24$ and total flower images are equal to 60, $80,100,120$ respectively the accuracy is $98.33 \%, 98.75 \%, 88.00 \%, 95.00 \%$ and times is $404.36 \mathrm{~s}, 533.66 \mathrm{~s}, 71.51 \mathrm{~s}, 6249.48 \mathrm{~s}$ with same learning rate.
Figure 11 shows the graph between NOI (number of images) with the average accuracy over the proposed algorithm.

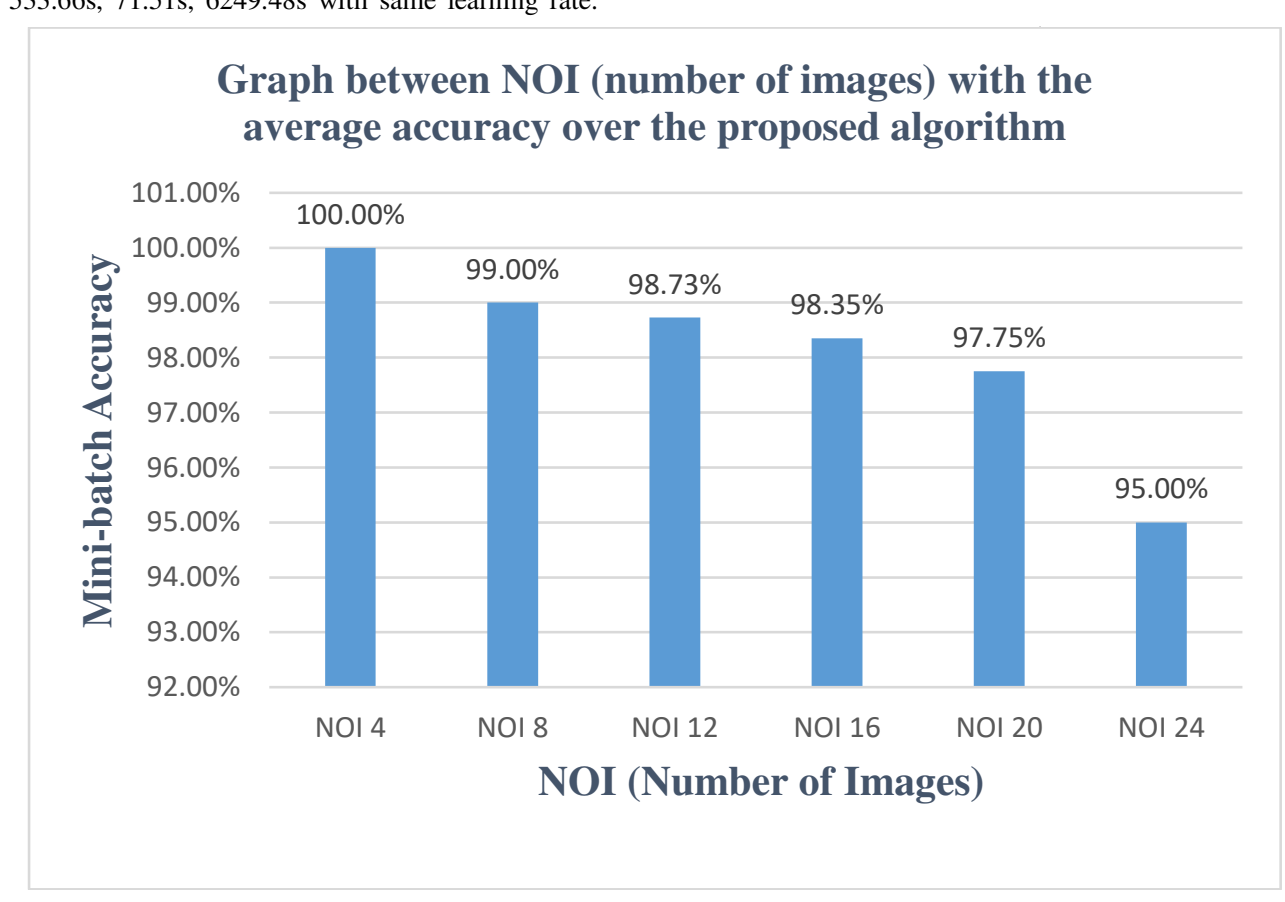

Figure 11: The graph between NOI (number of images) with the average accuracy over the proposed algorithm.

The proposed algorithm achieved an average accuracy of $95.84 \%$ and an average loss rate of 0.1837 . Figure 12 shows algorithm. graphically, the relation between NOI (number of images) and the average minimum-batch loss over the proposed 


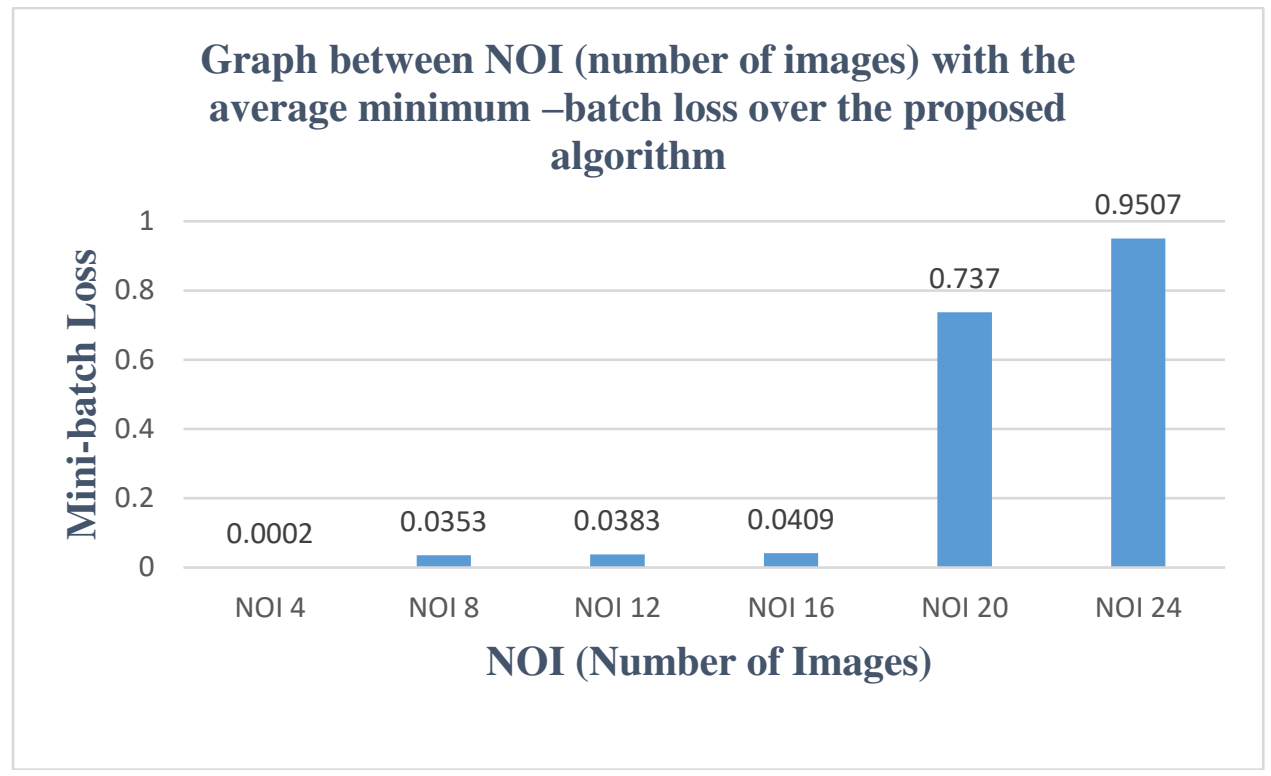

Figure 12: The graph between NOI (number of images) with the average minimum -batch loss over the proposed algorithm.

The percentage of wrong detection of flower is still high and needs further improvement. The proposed deep learningbased convolution neural network [32-48] provides best flower classification results on all datasets.

TABLE IV

FLOWER CLASSIFICATION RESULTS WITH TIME

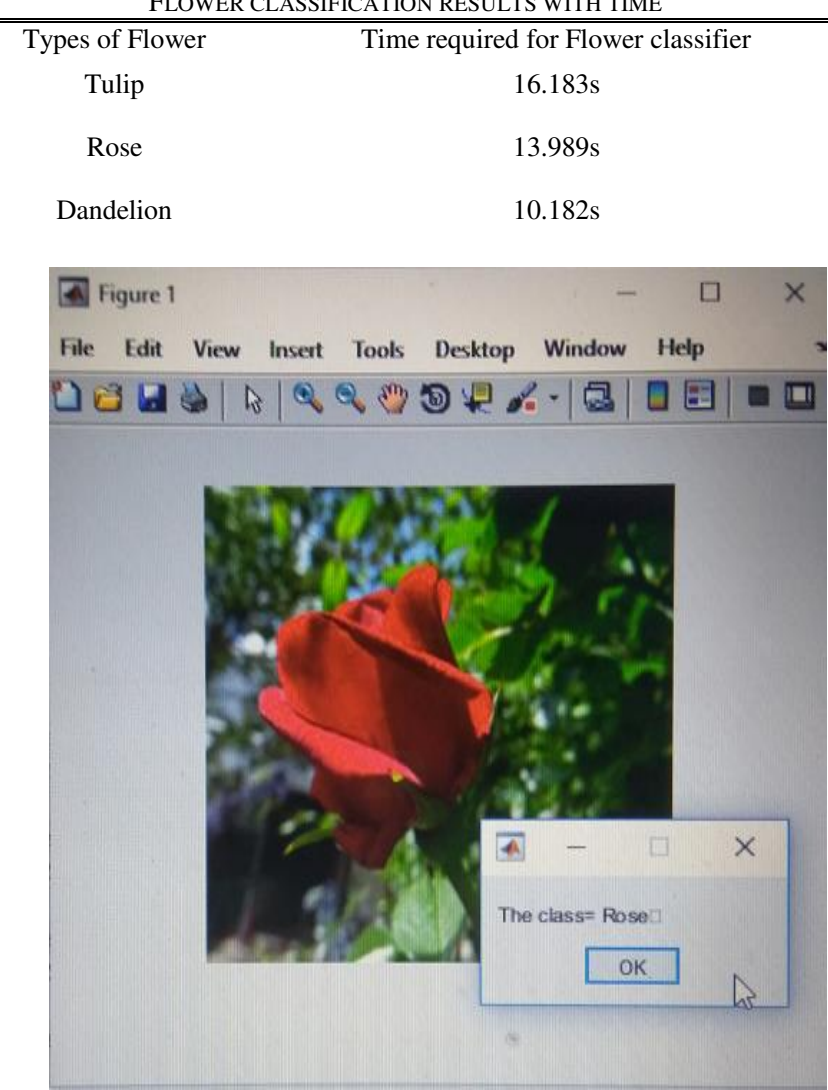

(a) Rose Detection
Daisy

$10.101 \mathrm{~s}$

Fully convolution neural networks is a rich class of models in which modern classification convolution neural network is a special case. Figure 13 (a, b, c, d) shows detected flower image which was downloaded from mathwork group during the experiment. Figure 13(a), 13(b), 13(c), 13(d) represented detection of Rose, Tulip, Dandelion, and Daisy flower image simultaneously.

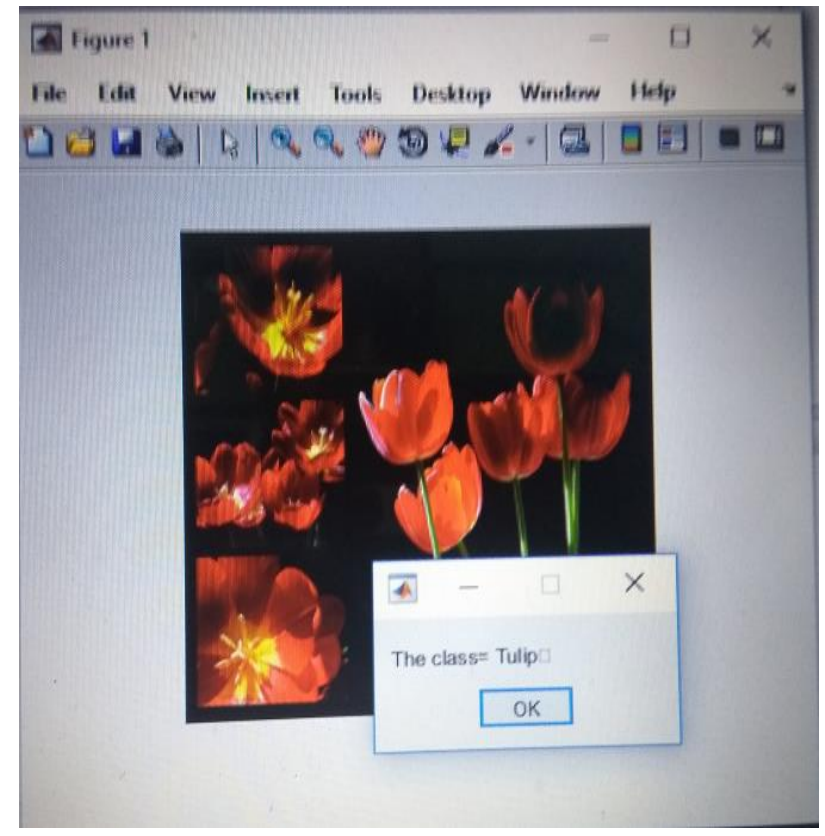

(b) Tulips detection 


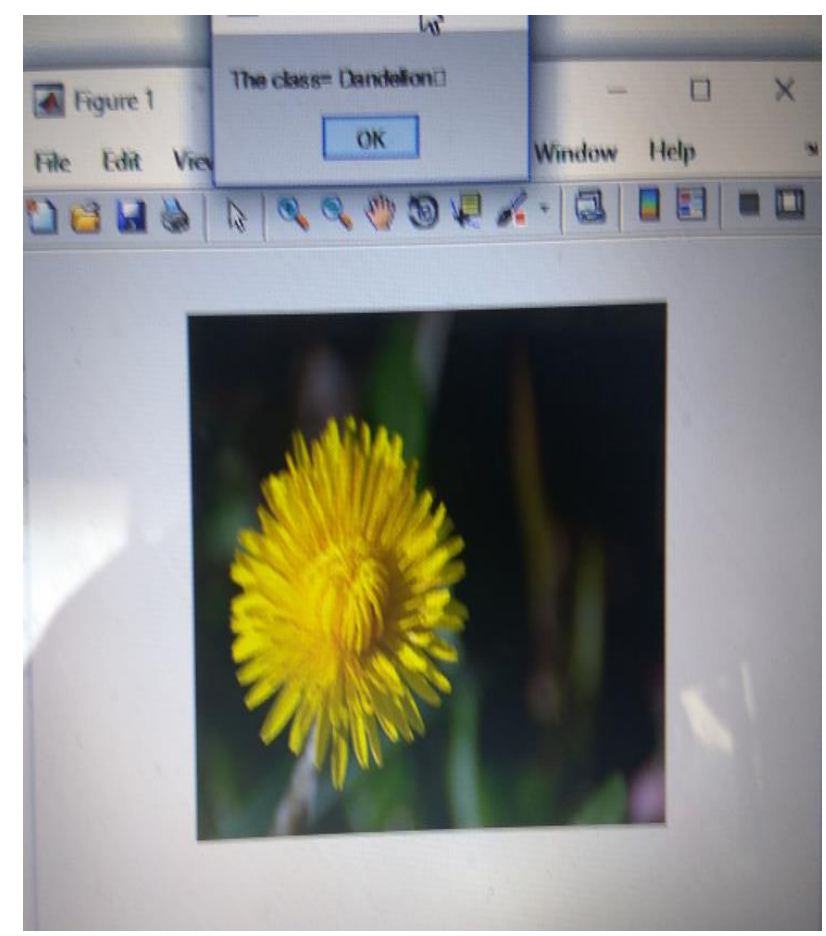

(c) Dandelion detection

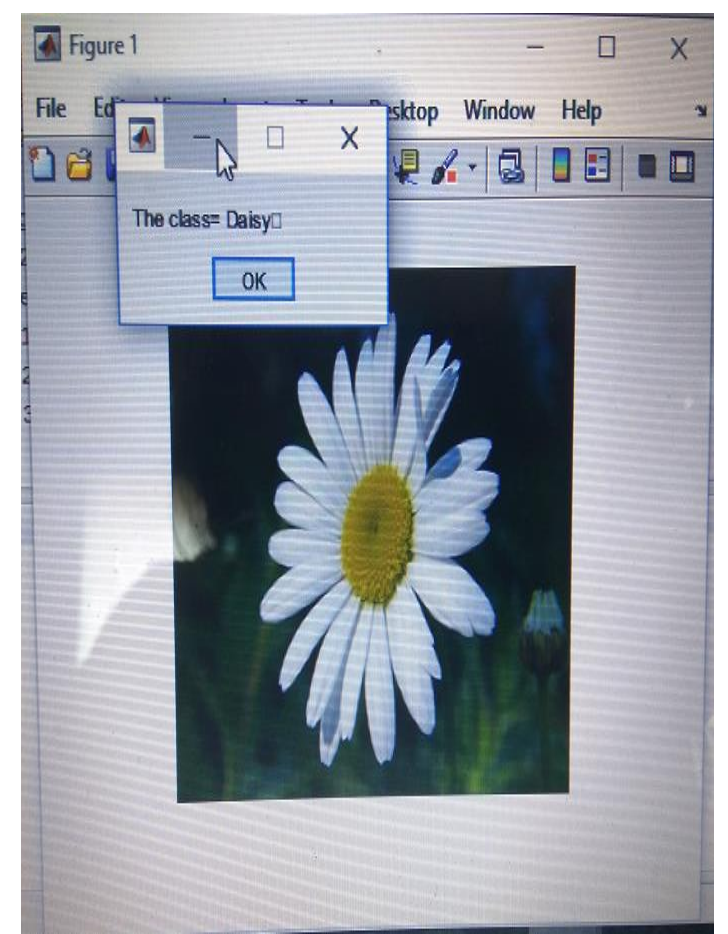

(d) Daisy Detection

Figure 13: Detected flower image.

\section{Results Analysis for experiment_2}

The training results are shown in Table 5. The columns represent the number of Epoch, iteration, time elapsed, loss, accuracy, and learning rate, used by proposing algorithm, respectively. Training was done on a single GPU with initializing flower image normalization by $64 * 64 * 3$.
The proposed algorithm experimented with different number of flower images and maximum epoch was 20. From the results of proposed method, it is clear that that when NOI = 1650 and epoch is equal to 20 , the time required for training is $55 \mathrm{~s}$ and accuracy is $98 \%$ with minimum batch loss equal to 0.4728 and learning rate is $1.00 \mathrm{e}-04$.

TABLE V

PERFORMANCES OF THE PROPOSED METHOD

\begin{tabular}{|c|c|c|c|c|c|}
\hline Epoch & Iteration & $\begin{array}{l}\text { Time Elapsed } \\
\text { (hh:mm:ss) }\end{array}$ & Mini-batch Loss & $\begin{array}{l}\text { Mini-batch } \\
\text { Accuracy }\end{array}$ & "Base-Learning Rate \\
\hline 1 & 1 & 00:00:00 & $10.00 \%$ & 9.9191 & $1.0000 \mathrm{e}-04$ \\
\hline 1 & 50 & 00:00:02 & $40.00 \%$ & 1.7673 & $1.0000 \mathrm{e}-04$ \\
\hline 1 & 100 & 00:00:03 & $30.00 \%$ & 0.8035 & $1.0000 \mathrm{e}-04$ \\
\hline 1 & 150 & 00:00:04 & $40.00 \%$ & 1.3736 & $1.0000 \mathrm{e}-04$ \\
\hline 2 & 200 & 00:00:05 & $50.00 \%$ & 1.2381 & $1.0000 \mathrm{e}-04$ \\
\hline 2 & 250 & 00:00:06 & $60.00 \%$ & 0.8936 & $1.0000 \mathrm{e}-04$ \\
\hline 2 & 300 & 00:00:07 & $40.00 \%$ & 1.5919 & $1.0000 \mathrm{e}-04$ \\
\hline 3 & 350 & 00:00:07 & $70.00 \%$ & 1.6425 & $1.0000 \mathrm{e}-04$ \\
\hline 3 & 400 & 00:00:08 & $70.00 \%$ & 0.7327 & $1.0000 \mathrm{e}-04$ \\
\hline 3 & 450 & 00:00:09 & $40.00 \%$ & 1.1009 & $1.0000 \mathrm{e}-04$ \\
\hline 4 & 500 & 00:00:10 & $70.00 \%$ & 1.3039 & $1.0000 \mathrm{e}-04$ \\
\hline 4 & 550 & 00:00:11 & $90.00 \%$ & 0.4999 & $1.0000 \mathrm{e}-04$ \\
\hline 4 & 600 & 00:00:12 & $40.00 \%$ & 0.9247 & $1.0000 \mathrm{e}-04$ \\
\hline 5 & 650 & 00:00:13 & $70.00 \%$ & 1.3738 & $1.0000 \mathrm{e}-04$ \\
\hline 5 & 700 & 00:00:14 & $100.00 \%$ & 0.2903 & $1.0000 \mathrm{e}-04$ \\
\hline 5 & 750 & $00: 00: 15$ & $50.00 \%$ & 0.8617 & $1.0000 \mathrm{e}-04$ \\
\hline
\end{tabular}




\begin{tabular}{|c|c|c|c|c|c|}
\hline 6 & 800 & 00:00:16 & $80.00 \%$ & 1.3198 & $1.0000 \mathrm{e}-04$ \\
\hline 6 & 850 & 00:00:17 & $100.00 \%$ & 0.4936 & $1.0000 \mathrm{e}-04$ \\
\hline 6 & 900 & 00:00:18 & $60.00 \%$ & 0.8758 & $1.0000 \mathrm{e}-04$ \\
\hline 7 & 950 & 00:00:19 & $100.00 \%$ & 1.2795 & $1.0000 \mathrm{e}-04$ \\
\hline 7 & 1000 & 00:00:20 & $100.00 \%$ & 0.3205 & $1.0000 \mathrm{e}-04$ \\
\hline 7 & 1050 & 00:00:21 & $90.00 \%$ & 0.8462 & $1.0000 \mathrm{e}-04$ \\
\hline 8 & 1100 & 00:00:22 & $100.00 \%$ & 1.2365 & $1.0000 \mathrm{e}-04$ \\
\hline 8 & 1150 & 00:00:23 & $90.00 \%$ & 0.3343 & $1.0000 \mathrm{e}-04$ \\
\hline 8 & 1200 & 00:00:24 & $90.00 \%$ & 0.8591 & $1.0000 \mathrm{e}-04$ \\
\hline 9 & 1250 & 00:00:24 & $100.00 \%$ & 1.3150 & $1.0000 \mathrm{e}-04$ \\
\hline 9 & 1300 & 00:00:25 & $90.00 \%$ & 0.2876 & $1.0000 \mathrm{e}-04$ \\
\hline 9 & 1350 & 00:00:26 & $100.00 \%$ & 0.7411 & $1.0000 \mathrm{e}-04$ \\
\hline 10 & 1400 & 00:00:27 & $100.00 \%$ & 1.1223 & $1.0000 \mathrm{e}-04$ \\
\hline 10 & 1450 & 00:00:28 & $60.00 \%$ & 0.2202 & $1.0000 \mathrm{e}-04$ \\
\hline 10 & 1500 & 00:00:29 & $60.00 \%$ & 0.9262 & $1.0000 \mathrm{e}-04$ \\
\hline 11 & 1550 & 00:00:30 & $100.00 \%$ & 1.2061 & $1.0000 \mathrm{e}-04$ \\
\hline 11 & 1600 & 00:00:31 & $80.00 \%$ & 0.2284 & $1.0000 \mathrm{e}-04$ \\
\hline 11 & 1650 & 00:00:32 & $40.00 \%$ & 0.7777 & $1.0000 \mathrm{e}-04$ \\
\hline 12 & 1700 & 00:00:33 & $100.00 \%$ & 1.6023 & $1.0000 \mathrm{e}-04$ \\
\hline 12 & 1750 & 00:00:33 & $60.00 \%$ & 0.2492 & $1.0000 \mathrm{e}-04$ \\
\hline 12 & 1800 & 00:00:34 & $80.00 \%$ & 0.6705 & $1.0000 \mathrm{e}-04$ \\
\hline 13 & 1850 & 00:00:35 & $80.00 \%$ & 1.2013 & $1.0000 \mathrm{e}-04$ \\
\hline 13 & 1900 & 00:00:36 & $60.00 \%$ & 0.1893 & $1.0000 \mathrm{e}-04$ \\
\hline 13 & 1950 & 00:00:37 & $80.00 \%$ & 0.7223 & $1.0000 \mathrm{e}-04$ \\
\hline 14 & 2000 & 00:00:38 & $100.00 \%$ & 1.1131 & $1.0000 \mathrm{e}-04$ \\
\hline 14 & 2050 & 00:00:39 & $40.00 \%$ & 0.2375 & $1.0000 \mathrm{e}-04$ \\
\hline 14 & 2100 & 00:00:40 & $60.00 \%$ & 0.7063 & $1.0000 \mathrm{e}-04$ \\
\hline 15 & 2150 & 00:00:41 & $100.00 \%$ & 1.1984 & $1.0000 \mathrm{e}-04$ \\
\hline 15 & 2200 & 00:00:41 & $60.00 \%$ & 0.1925 & $1.0000 \mathrm{e}-04$ \\
\hline 15 & 2250 & 00:00:42 & $60.00 \%$ & 0.6344 & $1.0000 \mathrm{e}-04$ \\
\hline 16 & 2300 & 00:00:43 & $100.00 \%$ & 1.1999 & $1.0000 \mathrm{e}-04$ \\
\hline 16 & 2350 & 00:00:44 & $80.00 \%$ & 0.1033 & $1.0000 \mathrm{e}-04$ \\
\hline 16 & 2400 & 00:00:45 & $80.00 \%$ & .5931 & $1.0000 \mathrm{e}-04$ \\
\hline 17 & 2450 & 00:00:46 & $100.00 \%$ & 1.1842 & $1.0000 \mathrm{e}-04$ \\
\hline 17 & 2500 & 00:00:47 & $80.00 \%$ & 0.1606 & $1.0000 \mathrm{e}-04$ \\
\hline 17 & 2550 & 00:00:47 & $80.00 \%$ & 0.5756 & $1.0000 \mathrm{e}-04$ \\
\hline 18 & 2600 & 00:00:48 & $100.00 \%$ & 1.0810 & $1.0000 \mathrm{e}-04$ \\
\hline 18 & 2650 & 00:00:49 & $80.00 \%$ & 0.0858 & $1.0000 \mathrm{e}-04$ \\
\hline 18 & 2700 & 00:00:50 & $80.00 \%$ & 0.5487 & $1.0000 \mathrm{e}-04$ \\
\hline 19 & 2750 & 00:00:51 & $100.00 \%$ & 1.0917 & $1.0000 \mathrm{e}-04$ \\
\hline 19 & 2800 & 00:00:52 & $90.00 \%$ & 0.0808 & $1.0000 \mathrm{e}-04$ \\
\hline
\end{tabular}


Columns in Table 5 shows the NOI (number of images of each type of flower and there are five types of flower images used by proposed algorithm for simulation), epoch, time elapsed, loss, accuracy, and learning rate, used by proposed algorithm, respectively. The training was done on a single GPU with initializing flower image normalization by $64 * 64 * 3$

The proposed algorithm experimented with different number of flower image and maximum epoch was 20. From the results of proposed method, it is clear that when NOI $=165$ and epoch is equal to 20 , then the time required for training is $00.55 \mathrm{~s}$ and accuracy up to be $98 \%$ with minimum batch loss equal to 0.4728 and learning rate is $1.00 \mathrm{e}-04$. The figure 14 gives a Training progress graph of the Accuracy and Loss with number of iteration over the proposed algorithm when run on GPU with NOI $=165$ of each class (number of images).
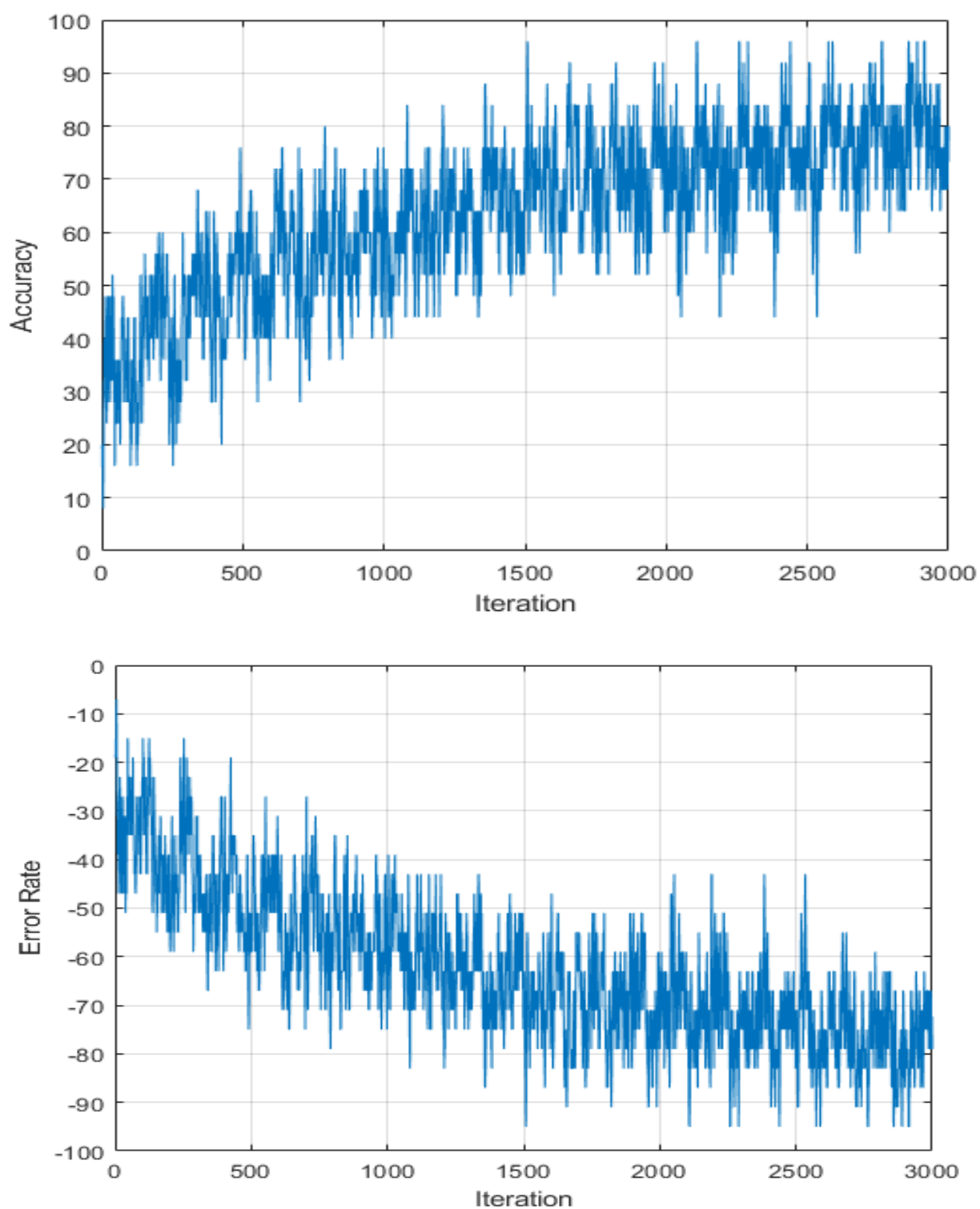

Figure 14: The Training progress graph of the Accuracy and Loss with number of Iteration over the proposed algorithm when run on GPU.

Table 6 shows the flower image detection time taken by proposed algorithm. When the proposed algorithm is executed on a high computation setup, it has an approximately $98 \%$ accuracy. This result compared with experiment_1 and it seems that the time required to detect the flower image by the proposed system is less when the same method runs on a high computation setup. 
TABLE VI

FLOWER CLASSIFICATION RESULTS WITH TIME

\begin{tabular}{ccc}
\hline $\begin{array}{c}\text { Types of } \\
\text { Flower }\end{array}$ & $\begin{array}{c}\text { Time required for } \\
\text { Flower classifier } \\
\text { (GPU) }\end{array}$ & $\begin{array}{c}\text { Time required for } \\
\text { Flower classifier } \\
\text { (CPU) }\end{array}$ \\
Tulip & $\begin{array}{c}\text { Experimnet_2 } \\
\text { E.987s }\end{array}$ & $\begin{array}{c}16.183 \mathrm{~s} \\
\text { Rose }\end{array}$ \\
Dandelion & $\mathbf{9 . 5 9 1 s}$ & $13.989 \mathrm{~s}$ \\
Daisy & $\mathbf{1 0 . 1 8 2 \mathrm { s }}$ & $10.182 \mathrm{~s}$ \\
& $\mathbf{5 . 7 8 5 s}$ & $10.101 \mathrm{~s}$ \\
\hline
\end{tabular}

\section{CONCLUSION}

An extended hybrid methodology based on deep learning CNN is proposed for the detection and segmentation of flower RGB images with $\mathrm{L}^{*} \mathrm{a} * \mathrm{~b}$ conversion. The experimental results show that the proposed methodology gives better accuracy and minimizes the error rate for RGB flower image segmentation.

- Experimental outcomes show that the accuracy of deep CNN depends on the weight function on the different layers if the value of weight is changed the learning accuracy is affected by the proposed method.

- The learning accuracy of deep convolution neural networks also depends on the Learning Rates, Batch Loss value, and types of images used for training deep convolution neural network.

- The accuracy is also affected by hardware configuration used by the implementation purpose.

The proposed deep learning-based convolution neural network provides the best flower classification results on all the datasets. There are various convolution neural network architectures like LeNet, AlexNet, GoogLeNet, VGGNet used to solve deep learning computer vision problems. In a future enhancement, the proposed system would be compared with a pertained network like LeNet, AlexNet, and GoogLeNet. The proposed system is applicable for Image Classification, Object Recognition, Content-Based Image retrieval system, Self-driving cars, Speech Recognition, etc.

\section{Declarations}

\section{Ethics approval and consent to participate}

Not applicable.

\section{Consent to publish}

Not applicable.

\section{Availability of data and materials}

The datasets generated during and/or analyzed during the current study are not publicly available, but are available from the corresponding author who was an organizer of the study.

\section{Competing interests}

The authors declare that they have no competing interests.

\section{Funding}

No Funding till date but we applied for in a government organization.

\section{Authors' contributions}

All of the authors contributed to the conception of the study, drafting and critical revision of the manuscript, and provided final approval of the manuscript.

\section{REFERENCES}

[1] Dhanalakshmi, S., and Dr T. Ravichandran. "A new method for image segmentation." International Journal of Advanced Research in Computer Science and Software Engineering 2, no. 9 (2012): 293-299.

[2] Tan, Y. J., K. S. Sim, and F. F. Ting. "Breast cancer detection using convolutional neural networks for mammogram imaging system." In 2017 International Conference on Robotics, Automation and Sciences (ICORAS), pp. 1-5. IEEE, 2017

[3] Baziyad M, Rabie T, Kamel I. Extending steganography payload capacity using the $1^{*} \mathrm{a}^{*} \mathrm{~b}^{*}$ color space. In2018 International Conference on Innovations in Information Technology (IIT) 2018 Nov 18 (pp. 1-6). IEEE.

[4] Baziyad, Mohammed, Tamer Rabie, and Ibrahim Kamel. "Extending steganography payload capacity using the $1^{*} \mathrm{a}^{*} \mathrm{~b}$ * color space." In 2018 International Conference on Innovations in Information Technology (IIT), pp. 1-6. IEEE, 2018.

[5] Chen, Yang, Won Suk Lee, Hao Gan, Natalia Peres, Clyde Fraisse Yanchao Zhang, and Yong He. "Strawberry yield prediction based on a deep neural network using high-resolution aerial orthoimages." Remote Sensing 11, no. 13 (2019): 1584

[6] Hiary, Hazem, Heba Saadeh, Maha Saadeh, and Mohammad Yaqub. "Flower classification using deep convolutional neural networks." IET Computer Vision 12, no. 6 (2018): 855-862.

[7] Gavai, Nitin R., Yashashree A. Jakhade, Seema A. Tribhuvan, and Rashmi Bhattad. "MobileNets for flower classification using TensorFlow." In 2017 International Conference on Big Data, IoT and Data Science (BID), pp. 154-158. IEEE, 2017.

[8] Xia, Xiaoling, Cui Xu, and Bing Nan. "Inception-v3 for flowe classification." In 2017 2nd International Conference on Image, Vision and Computing (ICIVC), pp. 783-787. IEEE, 2017.

[9] Gogul, I., and V. Sathiesh Kumar. "Flower species recognition system using convolution neural networks and transfer learning." In 2017 Fourth International Conference on Signal Processing, Communication and Networking (ICSCN), pp. 1-6. IEEE, 2017.

[10] Lodh, Avishikta, and Ranjan Parekh. "Flower recognition system based on color and GIST features." In 2017 Devices for Integrated Circuit (DevIC), pp. 790-794. IEEE, 2017

[11] Grauman, Kristen, and Trevor Darrell. "The pyramid match kernel: Discriminative classification with sets of image features." In Tenth IEEE International Conference on Computer Vision (ICCV'05) Volume 1, vol. 2, pp. 1458-1465. IEEE, 2005.

[12] Long, Jonathan, Evan Shelhamer, and Trevor Darrell. "Fully convolutional networks for semantic segmentation." In Proceedings of the IEEE conference on computer vision and pattern recognition, pp. 3431-3440. 2015.

[13] Girshick, Ross, Jeff Donahue, Trevor Darrell, and Jitendra Malik. "Rich feature hierarchies for accurate object detection and semantic segmentation." In Proceedings of the IEEE conference on computer vision and pattern recognition, pp. 580-587. 2014

[14] Greenspan, Hayit, Bram Van Ginneken, and Ronald M. Summers "Guest editorial deep learning in medical imaging: Overview and future promise of an exciting new technique." IEEE Transactions on Medical Imaging 35, no. 5 (2016): 1153-1159.

[15] Krizhevsky, Alex, Ilya Sutskever, and Geoffrey E. Hinton. "Imagenet classification with deep convolutional neural networks." In Advances in neural information processing systems, pp. 1097 1105. 2012.

[16] Dou, Qi, Hao Chen, Lequan Yu, Lei Zhao, Jing Qin, Defeng Wang, Vincent CT Mok, Lin Shi, and Pheng-Ann Heng. "Automatic detection of cerebral microbleeds from MR images via 3D convolutional neural networks." IEEE transactions on medical imaging 35, no. 5 (2016): 1182-1195

[17] Long, Jonathan, Evan Shelhamer, and Trevor Darrell. "Fully convolutional networks for semantic segmentation." In Proceeding of the IEEE conference on computer vision and pattern recognition, pp. 3431-3440. 2015.

[18] Li, Boxun, Yuzhi Wang, Yu Wang, Yiran Chen, and Huazhong Yang. "Training itself: Mixed-signal training acceleration for memristor-based neural network." In 2014 19th Asia and South Pacific Design Automation Conference (ASP-DAC), pp. 361-366 IEEE, 2014

[19] Ioffe, Sergey, and Christian Szegedy. "Batch normalization 
Accelerating deep network training by reducing internal covariate shift." arXiv preprint arXiv:1502.03167 (2015).

[20] Donahue, Jeff, Yangqing Jia, Oriol Vinyals, Judy Hoffman, Ning Zhang, Eric Tzeng, and Trevor Darrell. "Decaf: A deep convolutional activation feature for generic visual recognition." In International conference on machine learning, pp. 647-655. 2014.

[21] Zhang L, Lu L, Nogues I, Summers RM, Liu S, Yao J. DeepPap: deep convolutional networks for cervical cell classification. IEEE journal of biomedical and health informatics. 2017 May 19;21(6):1633-43

[22] Zhang, Ling, Le Lu, Isabella Nogues, Ronald M. Summers, Shaoxiong Liu, and Jianhua Yao. "DeepPap: deep convolutional networks for cervical cell classification." IEEE journal of biomedical and health informatics 21, no. 6 (2017): 1633-1643.

[23] Simonyan, Karen, and Andrew Zisserman. "Very deep convolutional networks for large-scale image recognition." arXiv preprint arXiv:1409.1556 (2014).

[24] Ronneberger, Olaf, Philipp Fischer, and Thomas Brox. "U-net: Convolutional networks for biomedical image segmentation." In International Conference on Medical image computing and computer-assisted intervention, pp. 234-241. Springer, Cham, 2015.

[25] Zhu, Xiaofeng, Heung-Il Suk, Li Wang, Seong-Whan Lee, Dinggang Shen, and Alzheimer's Disease Neuroimaging Initiative. "A novel relational regularization feature selection method for joint regression and classification in $\mathrm{AD}$ diagnosis." Medical image analysis 38 (2017): 205-214.

[26] Maji, Subhransu, Alexander C. Berg, and Jitendra Malik.

"Classification using intersection kernel support vector machines is efficient." In 2008 IEEE conference on computer vision and pattern recognition, pp. 1-8. IEEE, 2008.

[27] Ito, Satoshi, and Susumu Kubota. "Object classification using heterogeneous co-occurrence features." In European Conference on Computer Vision, pp. 701-714. Springer, Berlin, Heidelberg, 2010.

[28] Liu, Zeyu, Yantao Yang, and Qingdong Cai. "Neural network as a function approximator and its application in solving differential equations." Applied Mathematics and Mechanics 40, no. 2 (2019): 237-248.

[29] Donahue, Jeff, Yangqing Jia, Oriol Vinyals, Judy Hoffman, Ning Zhang, Eric Tzeng, and Trevor Darrell. "Decaf: A deep convolutional activation feature for generic visual recognition." In International conference on machine learning, pp. 647-655. 2014.

[30] Jaiswal, Varshali, Varsha Sharma, and Sunita Varma. "MMFO modified moth flame optimization algorithm for region based RGB color image segmentation." International Journal of Electrical \& Computer Engineering (2088-8708) 10 (2020).

[31] Rathore, Neeraj, and Inderveer Chana. "Load balancing and job migration techniques in grid: a survey of recent trends." Wireless personal communications 79, no. 3 (2014): 2089-2125.

[32] Rathore, Neeraj Kumar. "Ethical hacking \& security against cyber crime." Journal on Information Technology (JIT) 5, no. 1 (2016): 7 11.

[33] Rathore, Neeraj, and Inderveer Chana. "Job migration with fault tolerance based QoS scheduling using hash table functionality in social Grid computing." Journal of Intelligent \& Fuzzy Systems 27, no. 6 (2014): 2821-2833.

[34] Rathore NK, and Chana "Comparative analysis of checkpointing". In PIMR 3rd National IT conference, IT enabled practices and emerging management Paradigm book and category is communication technologies and security issues, Topic No/Name46, Prestige Management and Research, Indore, pp 32-35. 2008

[35] Rathore, Neeraj Kumar, and Inderveer Chana. "A cogitative analysis of load balancing technique with job migration in grid environment." In World congress on information and communication technology (WICT), Mumbai, IEEE proceedings paper, pp. 77-82. 2011.

[36] Rathore, Neeraj, and Inderveer Chana. "Variable threshold-based hierarchical load balancing technique in Grid." Engineering with computers 31, no. 3 (2015): 597-615

[37] Rathore, Neeraj, and Inderveer Chana. "A sender initiate based hierarchical load balancing technique for grid using variable threshold value." In 2013 IEEE International Conference on Signal Processing, Computing and Control (ISPCC), pp. 1-6. IEEE, 2013.

[38] Rathore, Neeraj Kumar. "Map reduce architecture for grid." $i$ manager's Journal on Software Engineering 10, no. 1 (2015): $21-$ 30.

[39] Rathore, Neeraj Kumar. "Efficient hierarchical load balancing technique based on grid." In 29th MP Young scientist congress, Bhopal, MP, p. 55.2014

[40] Rathore, Neeraj. "Performance of hybrid load balancing algorithm in distributed web server system." Wireless Personal Communications 101, no. 3 (2018): 1233-1246

[41] Rathore, Neeraj. "Efficient Agent Based Priority Scheduling and Load Balancing Using Fuzzy Logic in Grid Computing. i-manager's Journal on Computer Science, 3 (3) Sep-Nov 2015 Print ISSN 2347-2227." (2015): 2347-6141.

[42] Jain, Neelesh Kumar, Neeraj Kumar Rathore, and Amit Mishra "An efficient image forgery detection using biorthogonal wavele transform and improved relevance vector machine." Wireless Personal Communications 101, no. 4 (2018): 1983-2008.

[43] Rathore, Neeraj. "Dynamic threshold based load balancing algorithms." Wireless Personal Communications 91, no. 1 (2016) 151-185.

[44] Rathore, Neeraj Kumar, Umashankar Rawat, and Satish Chandra Kulhari. "Efficient hybrid load balancing algorithm." National Academy Science Letters 43, no. 2 (2020): 177-185.

[45] Jain, Neelesh Kumar, Neeraj Kumar Rathore, and Amit Mishra "An efficient image forgery detection using biorthogonal wavele transform and improved relevance vector machine." Wireless Personal Communications 101, no. 4 (2018): 1983-2008.

[46] Rathore, Neeraj Kumar, and Inderveer Chana. "Job migration policies for grid environment." Wireless Personal Communications 89, no. 1 (2016): 241-269.

[47] Sharma, Vishal, Rajesh Kumar, and Neeraj Rathore. "Topological Broadcasting Using Parameter Sensitivity-Based Logical Proximity Graphs in Coordinated Ground-Flying Ad Hoc Networks." J. Wirel. Mob. Networks Ubiquitous Comput. Dependable Appl. 6, no. 3 (2015): 54-72

[48] Rathore, Neeraj, and Inderveer Chana. "Report on hierarchal load balancing technique in grid environment." i-manager's Journal on Information Technology 2, no. 4 (2013): 21. 